\title{
Danish translation and linguistic validation of the BODY-Q: a description of the process
}

\author{
Lotte Poulsen $^{1} \cdot$ Michael Rose $^{2} \cdot$ Anne Klassen $^{3} \cdot$ Kirsten K. Roessler ${ }^{4}$. \\ Jens Ahm Sørensen ${ }^{5}$
}

Received: 6 June 2016 / Accepted: 20 August 2016 / Published online: 8 October 2016

(C) The Author(s) 2016. This article is published with open access at Springerlink.com

\begin{abstract}
Background Patient-reported outcome (PRO) instruments are increasingly being included in research and clinical practice to assess the patient point of view. Bariatric and body contouring surgery has the potential to improve or restore a patient's body image and health-related quality of life (HR-QOL). A new PRO instrument, called the BODY-Q, has recently been developed specifically for this patient group. The aim of the current study was to translate and perform a linguistic validation of the BODY-Q for use in Danish bariatric and body contouring patients.

Methods The translation was performed in accordance with the International Society For Pharmacoeconomics and Outcomes Research (ISPOR) and the World Health Organization (WHO) recommendations. Main steps taken included forward and backward translations, an expert panel meeting, and cognitive patient interviews. All translators aimed to conduct a conceptual translation rather than a literal translation and used a simple and clear formulation to create a translation understandable for all patients.
\end{abstract}

Lotte Poulsen

Lotte.poulsen2@rsyd.dk

1 Department of Plastic Surgery, Odense University Hospital, Sdr. Boulevard 29, 5000 Odense, Denmark

2 Department of Plastic Surgery, Hospital of Southwest Jutland, Esbjerg, Denmark

3 Department of Pediatrics, McMaster University, Hamilton, Canada

4 Department of Psychology, University of Southern Denmark, Odense, Denmark

5 Department of Plastic Surgery, Odense University Hospital, Odense, Denmark
Results The linguistic translation process led to a conceptually equivalent Danish version of the BODY-Q. The comparison between the back translation of the first Danish version and the original English version of the BODY-Q identified 18 items or instructions requiring re-translation. The expert panel helped to identify and resolve inadequate expressions and concepts of the translation. The panel identified 31 items or instructions that needed to be changed, while the cognitive interviews led to seven major revisions.

Conclusions The impact of weight loss methods such as bariatric surgery and body contouring surgery on patients' HRQOL would benefit from input from the patient perspective. A thorough translation and linguistic validation must be considered an essential step when implementing a PRO instrument to another language and/or culture. A combination of the ISPOR and WHO guidelines contributed to a straightforward and thorough translation methodology well suited for a Danish translation of the BODY-Q. The described method of translation and linguistic validation can be recommended for future translations of PRO instruments in the field of plastic surgery. Level of Evidence: Not ratable.

Keywords Translation · Cultural adaption · Linguistic validation $\cdot$ Patient-reported outcome $\cdot$ Bariatric surgery Body contouring surgery

\section{Introduction}

In recent years, there has been growing attention in health care concerning the evaluation of outcomes from the patient perspective. Well-developed, psychometrically sound, and clinically meaningful patient-reported outcome (PRO) instruments are increasingly being included in research and clinical practice to assess the patient point of view. Conventional methods to assess 
outcomes, such as mortality data, complications data, and photo review represent the health care provider perspective. Earlier reviews of PRO instruments for bariatric and/or body contouring surgery have called for the development of a new and comprehensive PRO instrument [1-5]. An international team recently followed internationally accepted guidelines and methods for the development of a new PRO instrument specific to measuring outcomes in weight loss and body contouring [6-11]. This new PRO instrument, called the BODY-Q, includes a set of 18 independently functioning scales and an obesity-specific symptom checklist. The scales measure three main concepts: appearance, healthrelated quality of life (HR-QOL), and experience of care $[12,13]$.

When questionnaires are adapted to another language and culture, it is extremely important to perform a proper translation and linguistic validation of the instrument. Well-developed PRO instruments achieve content validity through careful qualitative interviews, which only increases the importance of performing a careful translation. The International Society For Pharmacoeconomics and Outcomes Research (ISPOR) and the World Health Organization (WHO) have developed guidelines for good practice in the translation, linguistic validation, and cultural adaption process $[14,15]$. Following translation and linguistic validation for Danish patients, the BODY-Q could be used to support patient advocacy, patient education (e.g., satisfaction with information), and research efforts. Furthermore, using the BODY-Q scales in clinical practice will give patients the opportunity to report their concerns to their health care provider, who can use patients' answers in clinical decision-making [16]. Thus, the aim of the current study was to translate and perform a linguistic validation of the BODY-Q in Danish bariatric and body contouring patients.

Bariatric surgery, in combination with body contouring surgery, aims to improve or restore a patient's body image and HR-QOL. There have been many suggestions as to what constitutes the most important health concerns of the bariatric and body contouring surgery patient population, but little consensus on which questionnaires should be used to address these concerns [1-5]. A standardized approach to outcome assessment is needed, where only the most scientifically and clinically meaningful PRO instruments are used. Such an approach would advance knowledge about the impact of bariatric and body contouring surgery on patients and facilitate the ability to compare findings across studies and countries.

The BODY-Q $[12,13]$ addresses the need for a PRO instrument for weight loss and body contouring surgery patients $[17,18]$. The BODY-Q was developed following internationally recommended guidelines for item generation, item reduction, and psychometric evaluation [6-11]. The content for the BODY-Q was developed from a literature review, 63 qualitative patient interviews, 22 cognitive patient interviews and input from 9 experts. The BODY-Q measures three domains (appearance, HR-QOL, and experience of health care) via 18 independently functioning scales and an obesity-specific symptom checklist. The extensive qualitative steps were used to ensure that the scales are clinically grounded, relevant, and meaningful and capture the patient perspective $[12,13]$. The BODY-Q is unique from other PRO instruments in that it includes 10 scales to measure appearance-related concerns. These scales were created because appearance was found to be an important concern to patients undergoing weight loss and body contouring. In addition, concepts measured by the HR-QOL scales (body image and physical, psychological, social, and sexual functions) ask patients to answer "with their body in mind," ensuring the data are condition-specific. Each BODY-Q scale is independently functioning (no total scores) and scored from 0 (worse) to 100 (best) score. The BODY-Q can be used to monitor patients over their entire weight loss journey. Use of a modern psychometric method (i.e. Rasch [19]) means that the BODY-Q scales are well suited for use in both research and clinical practice. Rasch analysis allows for more accurate measurement, thus improving the BODYQ's ability to measure clinically meaningful change compared to previously suggested instruments $[12,13,20]$.

The aim of this study was to perform a Danish translation of The BODY-Q. Linguistic validation is the process to ensure that PRO concepts are equivalent and easily understood by people in countries not involved in the development. The translation was performed in accordance with the translation guidelines of ISPOR [14] and WHO [15]. The ISPOR guidelines describe principles of good practice for the translation and cultural adaptation process of PRO instruments and are, therefore, well suited for use in translating the BODY-Q. The WHO provides a framework with four steps i.e., forward translation, expert panel back translation, pre-testing, and cognitive interviewing and final version. The ISPOR guidelines define more refined steps i.e., preparation, forward translation, reconciliation, back translation, back translation review, harmonization, cognitive debriefing, review of cognitive debriefing results and finalization, proofreading, and final report.

\section{Material and methods}

We obtained permission to use the BODY-Q from the developers (Klassen et al., 2014). Ethical approval was applied from the Danish Ethics board prior to beginning the study. The translation process is described in Table 1. To begin, the project coordinators developed explanations for concepts measured by the BODY-Q and translators and expert panel participants were recruited. All translators aimed to create a conceptual translation rather than a literal translation and used a simple and clear formulation to create a translation that was understandable for all patients. The following six steps were taken:

1. Two independent forward translations were performed. A professional translator performed one, and a clinician with experience with the patient population performed the other. 
Both forward translators had Danish as their mother tongue and were fluent in English. A harmonization meeting between the two forward translators was held in order to achieve agreement on Danish version 1.

2. An independent professional translator produced a backward translation of the harmonized version. The translator had English as his mother tongue and was fluent in Danish. The back-translated version was compared with the original BODY-Q. All discrepancies were noted and discussed with the BODY-Q developers (Drs. Klassen and Pusic). Items from the back translation with different meaning than the English version were re-translated and shown to the developers. This process continued until a satisfactory result was achieved, leading to Danish version 2.

3. The translation team hosted an expert panel meeting. Prior to the meeting, the Danish version of the BODY-Q, along with translation guidelines, was sent to participants to review. The three translators, a specialist in bariatric surgery, and a specialist in body contouring surgery attended the meeting. All participants had Danish as their mother tongue and were fluent in English, except the back translator who had English as his mother tongue and was fluent in Danish. The aim of the meeting was to determine if the Danish version of the BODY-Q was understandable and measured all clinically relevant issues from the perspective of the clinicians. Feedback received was used to revise the scales, leading to consensus on Danish version 3 for pre-testing.

4. In the fourth step, 16 cognitive interviews were conducted with patients to determine if the BODY-Q instructions, response options, and items were clear, unambiguous, and relevant to respondents. Participants included six pre-bariatric (one patient was dyslectic), five pre-body contouring, and five post-body contouring. Patients were asked to read through the BODY-Q and discuss how they understood each item and the associated response options. Findings were used to make further adjustments to the translation, leading to Danish version 4.

5. In the fifth step, we conducted a further six cognitive interviews with patients with Danish version 4. Participants included two pre-bariatric, two pre-body contouring, and two post-body contouring. Findings were used to make further adjustments leading to Danish version 5 .

6 . In the last step, Danish version 5 was proofread independently by two clinicians, leading to the final Danish version of the BODY-Q.

\section{Results}

The translation process led to a Danish version of the BODY$\mathrm{Q}$ that was linguistically validated and conceptually equivalent to the original English version. An example of the major changes made throughout the translation process for one BODY-Q scale can be found in the Appendix.
In step 1, we found that the two independent forward translations had different views on the language, which required discussion in order to reach consensus. For some items, the clinician had included medical terms that the other translator thought would be challenging for patients to understand. However, the wordings of some items translated by the professional translator were judged to reflect insufficient knowledge of the patient group and journey. During the reconciliation and harmonization meeting, the two perspectives were found to provide complimentary information. Following discussion and revision, the two translators reached consensus on the Danish version 1.

The comparison of the back translation of Danish version 1 and the original English version with the developers of the BODY-Q identified 18 items or instructions where the meaning differed, requiring re-translation of the items and review by the developers. This iterative process was instrumental in helping to secure a conceptual as opposed to literal translation. For example, in the scale that measures body image, the original item "I feel positive towards my body" was initially back translated as "I have a positive relationship with my body," which was judged to have a different meaning and, thus, required re-translation. Another example was the use of the word "bothered" in the instructions of the scales measuring appearance of body contouring scars and excess skin. The word "bothered" proved difficult to translate into Danish, a challenge that has also previously been described [21]. The interactive process of comparing the Danish and English versions in an ongoing discussion with the developers helped to find consistent conceptual solutions.

The expert panel helped to identify and resolve unsatisfactory expressions and concepts in the back translation. Specifically, 31 items or instructions needed to be changed. For example, in the original version of the BODY-Q, the instructions for the patient experience scales that measure satisfaction with medical team included psychologists as a member of the medical team. Since psychologists are not part of medical teams for bariatric and/or body contouring care in Denmark, the expert panel decided to remove the word psychologist from the instructions. The expert panel meeting resulted in consensus on the Danish version 3 for cognitive debriefing.

Table 2 shows the characteristics of the participants included in the cognitive interviews. Patients were selected to ensure representation from all phases of the patient journey. Patients were debriefed about the study and asked to read systematically through the BODY-Q to identify problems (e.g. awkward or ambiguous wording) with the instructions, response options, and items and to suggest potential alternative wording. For example, some male patients pointed out that the Danish translation of "swimsuit" in the scales measuring the appearance of the abdomen and the body overall made them think of a female swimsuit. This finding was used to change the word for swimsuit to a gender-neutral word that retained the same meaning. 


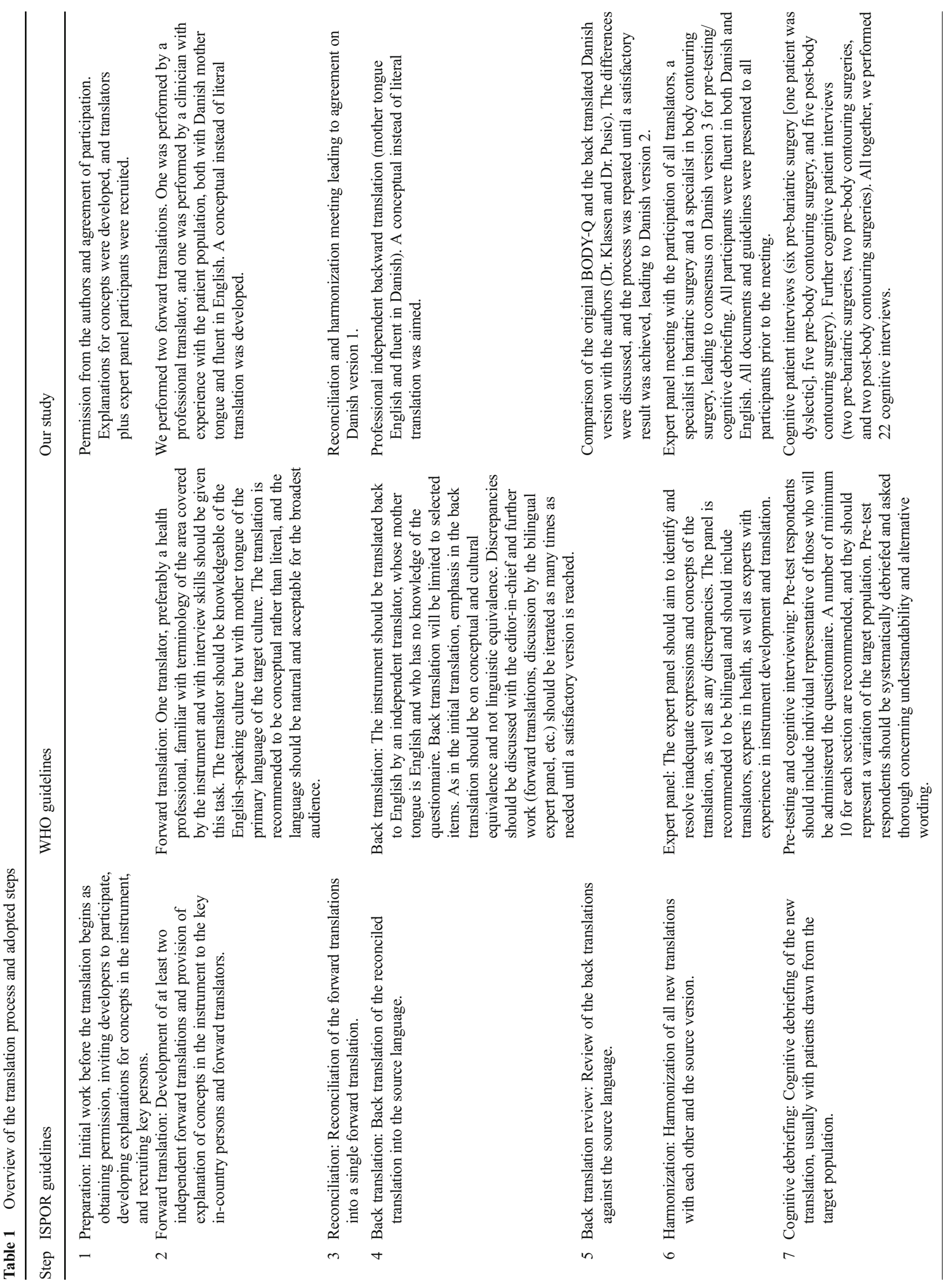




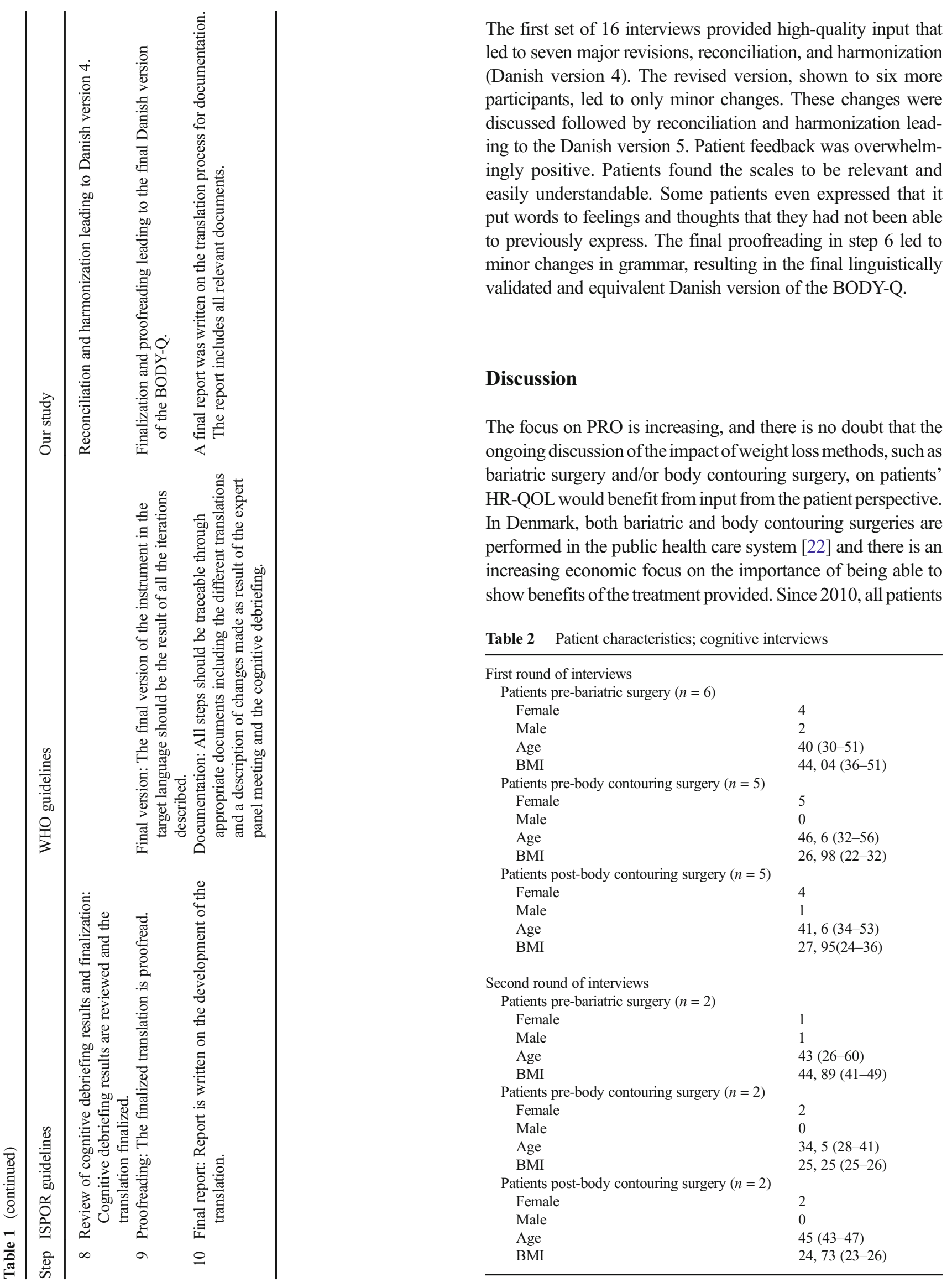


undergoing bariatric surgery have been registered in the Danish Bariatric Surgery Database [23]. The database has eight indicators, including complications data, weight loss data, and effect on comorbidity. This database (for both bariatric and body contouring patients) also measures HR-QOL using the Moorehead-Ardelt Quality of Life Questionnaire (MAQOL) [24]. The MAQOL is a 6-item scale that asks about self-esteem, physical, social, work, sexual, and eating behavior. This PRO instrument has important limitations in terms of content validity when used in body contouring patients, i.e., it does not ask about "appearance" even though weight loss following bariatric surgery can result in excess hanging skin that has a negative impact on body image and HR-QOL. Other important limitations of the MAQOL include a total score summing the six items, which has an ambiguous meaning; patients were not involved in its development (no qualitative interviews) [5-10]; and the scale was not designed to measure change across the entire weight loss journey. These limitations make the MAQOL an inappropriate tool to use as an indicator for quality monitoring and usefulness in the individual patient journey. Nonetheless, perhaps due to a lack of an appropriate PRO instrument, the MAQOL has been implemented in the Danish national database for bariatric and body contouring patients. The BODY-Q $[12,13]$ provides a comprehensive set of scales that could now be applied in the Danish bariatric and body contouring surgery patients. Strengths of the BODY-Q include that it was developed according to recommended guidelines for PRO instrument development [6-11], and it is clinical grounded, addressing relevant concerns of patients, including appearance concerns, HR-QOL, and experience of care. In the UK, the National Health Service (NHS) has recently recommend the use of select BODY-Q scales with all patients undergoing liposuction and abdominoplasty [25].

The ISPOR and WHO guidelines [14, 15] were adopted for the translation process, and a culturally adapted and equivalent Danish version of the BODY-Q was achieved. Using a combination of the two guidelines, we found the translation methodology to be straightforward, thorough and well suited for Danish translation of the BODY-Q. As previously described, there are a few differences between the ISPOR and WHO guidelines and both have strengths and limitations. For example, the ISPOR guidelines [14] recommend that two translators perform forward translation independently followed by a reconciliation meeting, whereas the WHO guidelines [15] emphasize the importance of achieving a conceptual rather than literal translation through the forward and back translations. The WHO guidelines explicitly recommend an expert panel. This step proved to be important in the translation of the BODY-Q into Danish and led to several crucial changes in both the wording of instructions and items. One could argue that we should also have included patients in the expert panel, but instead we conducted a large number of cognitive interviews and found that this approach was an acceptable way of ensuring that the patient voice was well represented. Furthermore, the WHO guideline includes a greater focus on the cognitive debriefing stage, which we also found to be of extreme importance. Feedback from patients was crucial and led to linguistic changes that improved the acceptability of the final scales. The strength of our cognitive interviewing was the number of patients we were able to include, as well as the ability to hand pick patients to ensure that different points on the weight loss journey were represented. A limitation in our sample was the smaller number of men interviewed (overall $18 \%$ ) compared to women (overall $81 \%)$. However, this difference reflects the distribution of gender in the bariatric and body contouring population [26].

Overall, we found that the combination of methods outlined by ISPOR and WHO provided a rigorous process that led to a high quality Danish translation of the BODY-Q. PRO instruments such as the BODY-Q are rapidly setting the standard for outcome measurement within the field of plastic and reconstructive surgery. The methods of translation and linguistic validation described here could be used to produce other translations of the BODY-Q, as well as other PRO instruments. Once a PRO instrument is rigorously translated and linguistically validated, it is important to ensure psychometric validation [20]. The next step of our research plan is to field test the BODY-Q in a large sample of bariatric and body contouring patients.

\section{Conclusion}

The translation and validation processes are an essential step in adapting a PRO instrument to another language and/or culture. We have translated the BODY-Q into Danish and tested its cultural relevance in a group of Danish patients undergoing bariatric and body contouring surgery. We found the translation methodology to be straightforward. The expert panel meeting and the cognitive debriefing were particularly useful steps taken to create a culturally equivalent translation. A thorough translation and a linguistic validation are of great importance when implementing a PRO instrument, and the described method of translation and linguistic validation can be recommended for future translations of PRO instruments in the field of plastic surgery.

\section{Compliance with ethical standards}

Ethical standards For this type of article formal consent from a local ethics committe is not required.

Conflict of interest Lotte Poulsen, Michael Rose, Kirsten K. Roessler, Jens Ahm Sørensen declare that they have no conflict of interest. The BODY-Q is owned by McMaster University and Memorial Sloan Kettering Cancer Centre. Anne Klassen is a codeveloper of the BODY$\mathrm{Q}$ and as such, will receive a share of any license revenues as royalties based on the inventor sharing policy.

Funding The study was funded by a grant from The Region of Southern Denmark and by the participating departments at Odense University Hospital and Hospital of Southwest Jutland. 


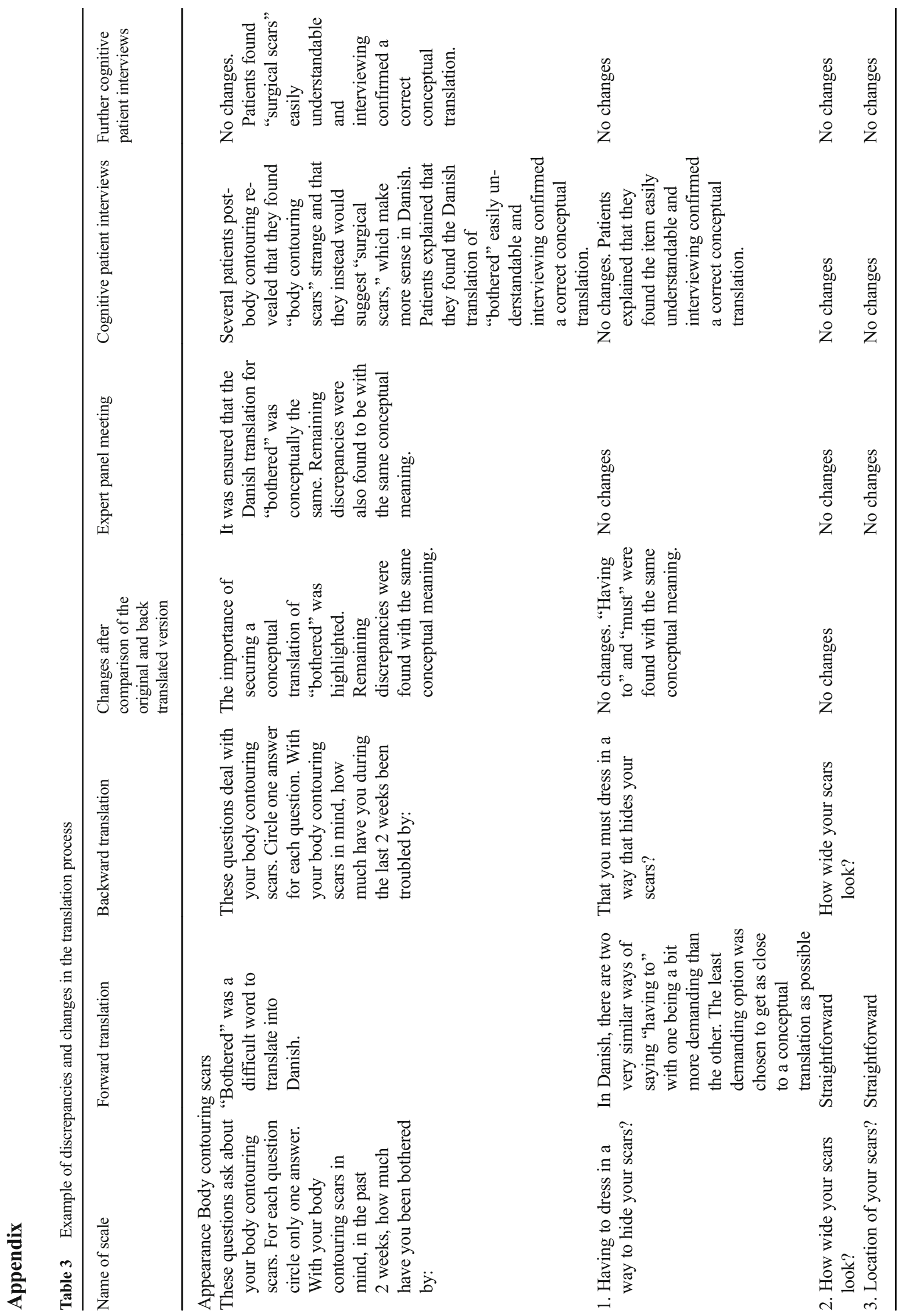




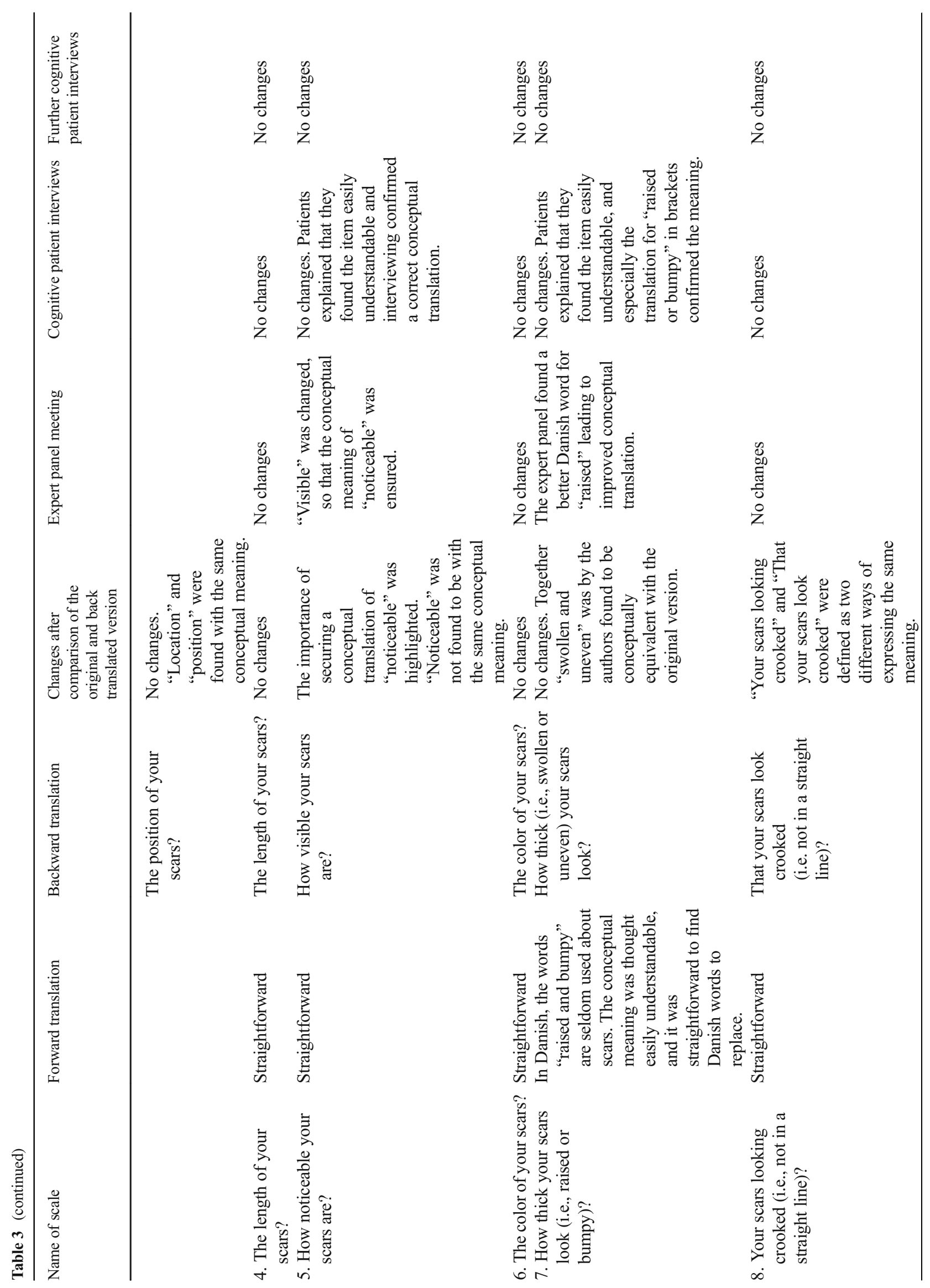


Open Access This article is distributed under the terms of the Creative Commons Attribution 4.0 International License (http:// creativecommons.org/licenses/by/4.0/), which permits unrestricted use, distribution, and reproduction in any medium, provided you give appropriate credit to the original author(s) and the source, provide a link to the Creative Commons license, and indicate if changes were made.

\section{References}

1. Tayyem R, Ali A, Atkinson J, et al. (2011) Analysis of healthrelated quality-of-life instruments measuring the impact of bariatric surgery: systematic review of the instruments used and their content validity. Patient 4:73-87

2. Reavey PL, Klassen AF, Cano SJ, et al. (2011) Measuring quality of life and patient satisfaction after body contouring: a systematic review of patient-reported outcome measures. Aesthet Surg J 31:807813

3. Jabir S. (2013) Assessing improvement in quality of life and patient satisfaction following body contouring surgery in patients with massive weight loss: a critical review of outcome measures employed. Plast Surg Int :515737.

4. Klassen A, Cano SJ, Scott A, et al. (2014) Assessing outcomes in body contouring. Clin Plast Surg 41:645-654

5. .Morley D, Jenkinson C, Fitzpatrick R (2013) A structured review of patient-reported outcome measures used in cosmetic surgical procedures. Oxford: Health Services Research Unit Department of Public Health University of Oxford.

6. Lasch KE, Marquis P, Vigneux M, et al. (2010) PRO development: rigorous qualitative research as the crucial foundation. Qual Life Res 19:1087-1096

7. Patrick DL, Burke LB, Gwaltney CJ, et al. (2011) Content validityestablishing and reporting the evidence in newly developed patientreported outcomes (PRO) instruments for medical product evaluation: ISPOR PRO good research practices task force report: part 1eliciting concepts for a new PRO instrument. Value Health 14:967977

8. Scientific Advisory Committee of the Medical Outcomes Trust (2002) Assessing health status and quality of life instruments: Attributes and review criteria. Qual Life Res 11:193-205

9. Cosmin (Consensus-based Standards for the selection of health Measurement Instruments) checklist. http://www.cosmin. nl/COSMIN\%20checklist.html. Accessed 12 Feb 2016

10. Mokkink LB, Terwee CB, Patrick DL, et al. (2010) The COSMIN study reached international consensus on taxonomy, terminology, and definitions of measurement properties for health-related patient-reported outcomes. J Clin Epidemiol. 63:737-745

11. Mokkink LB, Terwee CB, Patrick DL, et al. (2010) The COSMIN checklist for assessing the methodological quality of studies on measurement properties of health status measurement instruments: an international Delphi study. Qual Life Res. 18:539-549

12. Klassen A, Cano SJ, Scott A, et al. (2014) Assessing outcomes in body contouring. Clin Plast Surg. 41(4):645-654

13. Klassen AF, Cano SJ, Alderman A, et al (2016) The BODY-Q: A Patient-Reported Outcome Instrument for Weight Loss and Body Contouring Treatments. Plast Reconstr Surg Glob Open 13;4(4)

14. Wild D, Grove A, Martin M, et al. (2005) Principles of good practice for the translation and cultural adaptation process for patientreported outcomes (PRO) measures: report of the ISPOR task force for translation and cultural adaptation. Value Health. 8(2):94-104

15. WHO (World Health Organization). Process of translation and adaptation of instruments. http://www.who.int/substance abuse/research_tools/translation/en/. Accessed 12 Feb 2016 
16. Poulsen L, Klassen A, Jhanwar S, et al (2016) Patient Expectations of Bariatric and Body Contouring Surgery. Plast Reconstr Surg Glob Open 25;4(4):e694

17. Reavey PL, Klassen AF, Cano S, et al. (2011) Measuring qualityof-life and patient satisfaction after body contouring: a systematic review of patient-reported outcome measures. Aesthet Surg J 31: $807-813$

18. Klassen AF, Cano SJ, Scott A, et al. (2012) Satisfaction and qualityof-life issues in body contouring surgery patients: a qualitative study. Obes Surg 22:1527-1534

19. Rasch. Sample Size and Item Calibration [or Person Measure] Stability. http://www.rasch.org/rmt/rmt74m.htm. Accessed 12 Feb 2016

20. Hobart J, Cano S (2009) Improving the evaluation of therapeutic intervention in MS: the role of new psychometric methods. Health Technol Assess 13:1-200

21. Gawlicki MC, Brandt BA, McKown S, et al (2011) Application of the Bother Concept Across Cultures. Corporate Translations, Inc, East Hartford, CT, USA. Presented at the 18th Annual Conference of the International Society for Quality of Life Research, October 26-29; Denver, Colorado

22. Plastikkirurgisk korrektion efter massivt vægttab. Faglig visitationsretningslinje. København: Sundhedsstyrelsen, Sygehuse og Beredskab, 2013.

23. Dansk Fedmekirurgiregister, Årsrapporter 2011-2013. Kompetencecenter for Epidemiologi og Biostatistik (KCEB-) Nord. https://www.sundhed.dk/sundhedsfaglig/kvalitet/kliniskekvalitetsdatabaser/planlagt-kirugi/dansk-fedmekirurgi/ Accessed 12 February 2016.

24. Moorehead MK, Ardelt-Gattinger E, Lechner H, et al. (2003) The validation of the Moorehead-Ardelt quality of life Questionnarie II. Obesity Surg 13:684-692

25. Royal College of Surgeons. Patient Reported Outcome Measures. http://www.rcseng.ac.uk/surgeons/surgical-standards/workingpractices/cosmetic-surgery/datasets-and-qproms/patient-reportedoutcome-measures. Accessed 7 Sept 2016

26. Froylich D, Corcelles R, Daigle CR et al. Weight loss is higher among patients who undergo body contouring procedures after bariatric surgery. Surg Obes Relat Dis. 2015 Sep 21. 